\title{
CARACTERES Foliares EN DIEZ ESPECIES NEOTROPICALES Y argentinas de Canna (Cannaceae, Zingiberales)
}

\author{
MARÍA DE LAS MERCEDES CICIARELLI ${ }^{1,2}$, LILIAN M. PASSARELLI ${ }^{1}$ y \\ CRISTINA H. ROLLERI ${ }^{1}$
}

\begin{abstract}
Summary: Foliar characters in ten Neotropical and Argentine species of Canna (CannaceaeZingiberales). A study of epidermis and ontogeny of stomata of Neotropical species of Canna (Cannaceae) was performed using light and scanning electron microscopy. Leaf outlines, presence and types of indument, epicuticular wax distribution and types, epidermal patterns, characters of the paracytic adult stomata, were analyzed here in specimens of C. ascendens, C. coccinea, C. compacta, C. fuchsina, C. glauca, C. indica, C. lineata, C. paniculata, C. tandilensis and C. variegatifolia. The characters were correlated with the habitat of the plants. Ontogeny of stomata was studied on specimens of $C$. coccinea and C. glauca; both perigenous and mesogenous patterns of development occur in Canna, the latter not previously described for the family. Leaf characters turn out to be diagnostic in the specific level in the studied species of the genus; using them results important and very useful when the identification of species relays only on specimens in a vegetative condition.
\end{abstract}

Key words: Canna, Cannaceae, habitat, epidermis, wax, striae, stomatal ontogeny.

\begin{abstract}
Resumen: Se llevó a cabo un estudio con microscopía de luz y electrónica de barrido (MEB) de los caracteres de la epidermis y de la ontogenia de los estomas en especies neotropicales de Canna (Cannaceae). Se estudiaron la presencia y tipos de indumento, el contorno foliar, los tipos de cera epicuticular, los modelos epidérmicos, el ordenamiento celular típico de epifilos e hipofilos, el espesor de la pared externa de las células epidérmicas y los caracteres de los estomas adultos paracíticos en ejemplares de C. ascendens, C. glauca, C. indica, C. lineata, C. paniculata, C. tandilensis y C. variegatifolia. Los caracteres se correlacionaron con el hábitat de las plantas. La ontogenia estomática se analizó en dos especies: $C$. coccinea y $C$. glauca; se encontraron desarrollos perígenos y mesógenos, éstos no descritos previamente para la familia. El conjunto de los caracteres foliares macroscópicos y los microscópicos resultan diagnósticos en las especies analizadas y por ello, se consideran especialmente útiles en la determinación de especies de Canna cuando se trabaja con ejemplares en estado vegetativo y $\sin$ flores.
\end{abstract}

Palabras clave: Canna, Cannaceae, hábitat, epidermis, ceras, estrías, ontogenia de estomas.

\section{INTRODUCCIÓN}

Las Cannaceae Link constituyen una familia de plantas herbáceas, rizomatosas, perennes, nativas de América tropical y subtropical, pertenecientes

\footnotetext{
${ }^{1}$ Laboratorio de Estudios de Anatomía Vegetal Evolutiva y Sistemática (LEAVES), Facultad de Ciencias Naturales y Museo, Universidad Nacional de La Plata, $64 \mathrm{~N}^{\circ} 3$ entre 120 y diagonal 113, B1904 DZB, La Plata Argentina.

${ }^{2}$ Correspondencia autor: María de las Mercedes Ciciarelli, mercedes.ciciarelli@yahoo.com.ar; mercedes.ciciar@ gmail.com
}

al orden Zingiberales Grisebach. Se caracterizan por sus hojas grandes, verdes, verde rojizas o glaucas y por las flores, generalmente llamativas por su tamaño y color. El único género, Canna L., reúne unas veinticinco especies conocidas con diferentes nombres vulgares (achiras, adeiras, cañas de Indias). En la Argentina crecen unas dieciséis especies (Ciciarelli \& Rolleri, 2008; Ciciarelli, 2015), en el noroeste y noreste del país, en selvas lluviosas, de neblina y selvas en galería y también están presentes en las fitocenosis de áreas inundables ribereñas y humedales rioplatenses (Passarelli et al., 2014). 
Las ceras vegetales han sido estudiadas desde enfoques diversos, desde los más generales de Eglinton et al. (1962), Wilkinson (1979), Baker (1982), Bianchi (1995), Von Wettstein-Knowles (1995), Barthlott et al. (1998), Holloway \& Jeffree (2005) y Jeffree (2006), hasta otros más específicos o relacionados géneros de Zingiberales $s$. l., como los de Freeman \& Turner (1985), Arrieche \& Sanabria (1995), Martins et al. (2010) y Meusel et al. (1994). Neinhuis \& Barthlott (1997), analizaron entre otras, las ceras epicuticulares de Canna glauca L., a la que describieron con el aspecto de pequeñas placas (platelets) y consideraron la epidermis de esa especie como papilosa, en el contexto de un estudio funcional de las ceras como cubiertas repelentes de agua en plantas tropicales. Más recientemente, Ciciarelli (2007), observó con MEB describió e ilustró los tipos de ceras epicuticulares, estrías cuticulares, modelos epidérmicos y estomas adultos en $C$. ascendens Ciciar. y C. variegatifolia Ciciar., y llamó escamas a esos tipos morfológicos de ceras epicuticulares. Ciciarelli et al. (2010), estudiaron con MEB e ilustraron los caracteres epidérmicos de $C$. coccinea Miller, C. fuchsina Ciciar. y $C$. glauca, dando a conocer también los tipos de ceras epicuticulares, estrías y modelos epidérmicos y estomas adultos en estas especies; además Ciciarelli efectuó similares observaciones sobre $C$. lineata Ciciar. (2014) y C. tandilensis Ciciar. (2015).

Los primeros estudios sobre epidermis y anatomía foliar en especies de Canna aparecen hacia fines del siglo XIX, en obras generales sobre anatomía de Monocotiledóneas, como las de Falkenberg (1876), Leblois (1887), Lutz (1897) y Lov (1926) y, posteriormente, las de Solereder \& Meyer (1930), Stebbins \& Khush (1961) y Tomlinson $(1961,1962$, 1969, 1974). Stebbins \& Khush (1961), llevaron a cabo una amplia prospección sobre morfología de estomas en Monocotiledóneas e incluyeron géneros de varias familias de Zingiberales, entre ellas Cannaceae, Musaceae Juss. y Zingiberaceae Lindley, en la que utilizaron el término complejo estomático, propuesto previamente por Strasburger (1866) y Tognini (1897), en lugar de estoma. Stebbins \& Khush (1961), no ilustraron el complejo estomático de las Cannaceae pero lo incluyeron en la categoría de estomas formados por dos células oclusivas y dos anexas dispuestas lateralmente respecto de aquéllas. Tomlinson (1969), estudió la morfología foliar en Zingiberales y fue el primer autor que mencionó la alternancia de filas celulares con elementos de diferente morfología en la epidermis de $C$. indica L. Tomlinson (1974), también utilizó esa especie como modelo para analizar la estomatogénesis de Zingiberales y proponer un nuevo conjunto de términos para Monocotiledóneas, basado en los planos de las divisiones que tienen lugar en las células protodérmicas residuales que darán origen a los estomas. Olatunji (1980) estudió los tipos de estomas adultos de $C$. indica y describió para esa especie estomatogénesis de tipo perígeno, un término propuesto previamente por Pant (1965). Posteriormente Wilkinson (1979), aplicó al estoma adulto descrito por Stebbins \& Khush (1961), el nombre de paracítico, un término original de Vesque (1889), que es el que ha prevalecido hasta el momento. En relación con la ontogenia, los escasos trabajos que trataron el desarrollo de estomas de Monocotiledóneas en general los consideraron perígenos (Pant 1965), pero obras más recientes, como las de Payne (1979), Rasmussen (1981), Baranova (1992) y Croxdale (1998), entre otras, aceptaron la secuencia mesógena como un modelo de ontogenia también presente en el grupo.

Aquí se presenta un análisis de la morfología foliar de diez especies neotropicales y argentinas de Canna: C. ascendens, C. coccinea, C. compacta Rosc., C. fuchsina, C. glauca, C. indica, C. lineata, C. paniculata Ruiz et Pav., C. tandilensis y $C$. variegatifolia. Con excepción de C. compacta, un elemento presente en las Yungas del NO de la Argentina, todas crecen en selvas lluviosas y selvas en galería del NE del país y en numerosos humedales rioplatenses.

Este trabajo tiene por objetivos reunir y actualizar la información sobre Cannaceae, dispersa en trabajos parciales previos de las autoras (Ciciarelli, 2007, 2014, 2015; Ciciarelli \& Rolleri, 2008; Ciciarelli et al., 2010), ampliar el conocimiento de la familia y especialmente, analizar el potencial valor diagnóstico de un conjunto de caracteres foliares externos en las especies en relación con la ecología, con el fin de determinarlas en ausencia de caracteres florales.

\section{Materiales y Métodos}

Se utilizó material fresco y herborizado. El material fresco fue recolectado en las provincias 


\section{Ciciarelli et al. - Caracteres foliares Canna (Cannaceae)}

de Salta, Entre Ríos, Córdoba, Santa Fe y Buenos Aires. El herborizado proviene de los siguientes herbarios: del Instituto de Botánica del Nordeste (CTES), del Instituto-Fundación Miguel Lillo (LIL), de la Facultad de Ciencias Naturales y Museo de La Plata (LP) y del Instituto de Botánica Darwinion (SI). El material estudiado se puede consultar en el Apéndice 1.

Para estudios de epidermis se aclararon trozos de hojas con hidróxido de sodio al 3\% durante 2 días y se completó el proceso con hipoclorito de sodio comercial diluido 1:1. Para preparaciones definitivas se aplicaron diversas coloraciones: safranina $1 \%$ en alcohol absoluto-xilol (1:1), safranina-fast-green $2 \%$ en metil-cellosolve (Gurr, 1966), fast-green $1 \%$ en alcohol $95 \%$ y coloración de Foster (1949). Las hojas juveniles se fijaron en Carnoy (Johansen, 1940) y se colorearon con azul de Toluidina.

Para estudios con MEB, trozos de hipofilo y epifilo de hojas sin tratar, de $3 \times 3 \mathrm{~mm}$, se montaron sobre cinta adhesiva de doble faz y se metalizaron al vacío en un metalizador JEOL JFC 1100. Las fotografías se tomaron con un microscopio electrónico Jeol JFC T100 en el Servicio de Microscopía Electrónica de la Facultad de Ciencias Naturales y Museo (Universidad Nacional de La Plata, Argentina).

Los modelos epidérmicos se analizaron en hojas basales, medias y apicales, pero se ilustran sólo los de las hojas medias. Las dimensiones y densidad de los estomas se tomaron en hojas medias de todos los ejemplares estudiados; las dimensiones se expresan en largo $\mathrm{x}$ ancho en $\mu \mathrm{m}$ y la densidad en número de estomas por $\mathrm{mm}^{2}$. Los valores dados representan el promedio de 25 medidas por muestra.

Los términos de contorno foliar y modelos epidérmicos se utilizaron en otros trabajos de la autora (Ciciarelli, 2007, 2014, 2015, Ciciarelli et al., 2010). Para describir la ontogenia de estomas se utilizaron los términos descriptivos células de contacto (Tomlinson, 1974), equivalente al de anexas perígenas (Pant, 1965); mientras que para los estomas adultos se siguió a Wilkinson (1979). Los términos aplicados al indumento siguen a Theobald et al. (1979) y los relacionados con las ceras epicuticulares se encuentran en Wilkinson (1979) o bien son descriptivos y corresponden a las autoras.

\section{Resultados}

Habitat. En hábitats naturales, las especies de Canna crecen como elementos anfibios o terrestres $\mathrm{y}$, en este caso en suelos húmedos a muy húmedos, en selvas lluviosas, zonas de humedales o selvas en galería. Canna glauca y C. fuchsina son anfibias, ocasionalmente se adaptan como anfibias o palustres facultativas y toleran alta exposición solar en zonas de clima templado húmedo. Canna ascendens, $C$. lineata, $C$. tandilensis y $C$. variegatifolia son riparias, frecuentes en humedales, en suelos moderadamente encharcados; como las anfibias, suelen ser tolerantes a la exposición solar. Canna coccinea, C. compacta y $C$. paniculata son especies de selvas lluviosas bajas o de altura, crecen a la sombra pero pueden adaptarse como elementos terrestres higrófilos en áreas subselváticas y bordes de selvas. En este grupo se incluye $C$. indica, probablemente la más ubicua en cuanto a exigencias ecológicas, ya que puede adaptarse a vivir como una mesófita en zonas de clima templado húmedo, aunque siempre con requerimientos de suelos húmedos, bien drenados.

Contorno foliar. Las especies anfibias como $C$. glauca y $C$. fuchsina, y algunas riparias o de suelos húmedos a moderadamente inundables, como $C$. lineata y $C$. tandilensis tienen láminas foliares relativamente angostas: el contorno es linear a linearlanceolado, con una relación 1/a de 6:1 en C. glauca y lanceolado angosto con una relación 1/a de 3:1 en C. fuchsina y de 4-5:1 en C. lineata y $C$. tandilensis. Canna ascendens y $C$. variegatifolia, ambas riparias, tienen hojas más anchas, con el contorno lanceolado a elíptico y con una relación 1/a de 3-4:1.

Los higrófitos terrestres, de selvas lluviosas y selvas de altura, como C. compacta, C. coccinea, $C$. indica y $C$. paniculata, son comparativamente latifoliados. El contorno foliar es elíptico a oblongo en $C$. compacta y elíptico en $C$. indica, en ambas con una relación 1/a de 2-3:1, ovado-oblongo en $C$. coccínea con una relación $1 /$ a de $2: 1$ y lanceolado en C. paniculata, con una relación 1/a de 3-4:1.

Indumento. Se encuentra exclusivamente en el hipofilo, es piloso, de tipo lanuginoso con el aspecto de una pubescencia densa formada por pelos largos, enmarañados, ondulados o rizados, con paredes delgadas, distribuidos sobre lámina y venas; también se observan escamas y papilas. Se presenta en dos especies: C. compacta y C. paniculata.

En C. compacta el indumento consiste solamente 
de pelos 1-celulares, formados por una célula única extremadamente larga, con paredes delgadas, inserta entre otras epidérmicas.

En $C$. paniculata se encuentran pelos, escamas y además, abundantes papilas epidérmicas. Los pelos son variados: hay pelos 1-celulares, similares a los de C. compacta pero más cortos, pelos 3-5-celulares con paredes engrosadas y pelos pluricelulares $1-2$ veces ramificados, estrellados desde la base o bi-estrellados, con una base 1-celular cilíndrica, un cuerpo 1-celular formado por una célula bifurcada en la que cada bifurcación lleva una o varias células cilíndricas, formando así un pelo doblemente estrellado. Las escamas son basifijas, discoloras, lanceoladas, de hasta $5 \mathrm{~mm}$, con ápice hialino largamente acuminado, márgenes translúcidos irregularmente denticulados y cuerpo de color castaño rojizo con un área central oscura, formada por células con paredes engrosadas.

Células Epidérmicas propiamente dichas. En todas las especies hay células con paredes externas engrosadas, cutinizadas, estriadas y cubiertas por ceras que se disponen de manera diversa en cuanto a distribución y densidad.

Ceras. Las ceras tienen el aspecto de escamas o laminillas discretas o bien se distribuyen en placas muy delgadas que se dispersan al azar y a veces se levantan desde la superficie en forma de escamas irregulares que pueden ser grandes. Las escamas o laminillas discretas se disponen densamente y son las más pequeñas en hipofilos y epifilos de $C$. glauca (Fig. 1 A-B), aparecen, también densamente agrupadas, en áreas discontinuas sobre la pared externa de las epidérmicas como en $C$. fuchsina (Fig. $1 \mathrm{C}$ ) y $C$. tandilensis (Fig. 2 E), pueden predominar en las paredes de contacto celulares y en masas superficiales poco extendidas como en C. ascendens, C. coccinea, C. compacta y C. variegatifolia (Figs. 1 D-F y 2 A-B). La distribución no es igual en epifilos e hipofilos y es más abundante en estos últimos. Rara vez se encuentra cera sobre las oclusivas de los estomas pero sí puede cubrir la pared externa de sus anexas, como ocurre en $C$. lineata y $C$. fuchsina (Figs. 2 y 3 C-D), en esta especie con variaciones, ya que se presentan anexas con y sin cobertura cerosa. Canna paniculata tiene escasa cera en los hipofilos pero una cubierta continua y delgada en epifilos, que en algunos casos incluye cera granular. Las placas delgadas que se desprenden o levantan en escamas irregulares aisladas se encontraron en $C$. indica (Fig. $2 \mathrm{~F}$ ).

Estrías. Las estrías cuticulares son frecuentes en casi todas las especies, excepto en $C$. indica. Se encuentran en epifilos e hipofilos. En general son gruesas, con lomos curvos, irregulares y no siempre alineadas, en C. ascendens, $C$. glauca, $C$. paniculata y C. variegatifolia (Fig. $1 \mathrm{D}-\mathrm{F}$ ); mientras que, son finas, delgadas, bastante regulares y a veces alineadas en forma casi paralela en C. coccinea, C. fuchsina, $C$. lineata y C. tandilensis (Fig. 2 A).

Modelos epidérmicos. Las células epidérmicas en vista superficial tienen un patrón poligonal a subpoligonal, con paredes rectas a levemente curvas. En los hipofilos las paredes celulares externas están notablemente engrosadas, con espesores promedio de 3-10 $\mu \mathrm{m}$. Los mayores espesores, de hasta $10 \mu \mathrm{m}$, se observaron en $C$. glauca, se midieron espesores de hasta $8 \mu \mathrm{m}$ en $C$. coccinea y $C$. indica, de hasta 6 $\mu \mathrm{m}$ en $C$. ascendens y en $C$. variegatifolia, de hasta 5 $\mu \mathrm{m}$ en $C$. compacta, $C$. lineata y $C$. tandilensis y de 4-6 $\mu \mathrm{m}$ en $C$. fuchsina; solamente en $C$. paniculata las paredes celulares son algo más delgadas, con un espesor del orden de los $3 \mu \mathrm{m}$.

Las células epidérmicas se disponen en hileras ordenadas, tanto en epifilos como en hipofilos y se observan hileras sin estomas e hileras interrumpidas con frecuencia regular por los estomas y sus anexas o bien, según el área de la hoja, por las protodérmicas que darán origen a los estomas. El modelo poligonal de las hileras puede variar en relación con los diámetros celulares.

Se encuentran hileras sin estomas formadas por células isodiamétricas o con una relación 1/a no mayor de 2:1 en epifilos e hipofilos de $C$. compacta, $C$. glauca (Fig. 3 A), C. fuchsina y C. lineata (Fig. 3 C-D, F), e hipofilos de $C$. indica (Fig. 3 B) у C. tandilensis (Fig. 3 E).

Se presentan hileras sin estomas formadas por células poligonales subrectangulares, longitudinalmente más largas que anchas, con una relación 1/a mayor de 3:1 y hasta de 6:1, en hipofilos de C. indica (Fig. 4 A), C. coccinea (Fig. 4 B), C. paniculata (Fig. 4 E), C. ascendens (Fig. 4 D), C. lineata (Fig. 4 C), C. tandilensis y C. variegatifolia (Fig. 4 F). Las células de estas hileras tienen pared externa lisa o más frecuentemente, estriada; las estrías son escasas y erráticas, dispersas a ausentes an áreas amplias de la epidermis en $C$. indica, pero son muy marcadas en las restantes especies, a veces anchas como en C. ascendens, C. coccinea, C. glauca, C. paniculata, C. variegatifolia o muy finas, largas como toda la longitud de las células y próximas, 

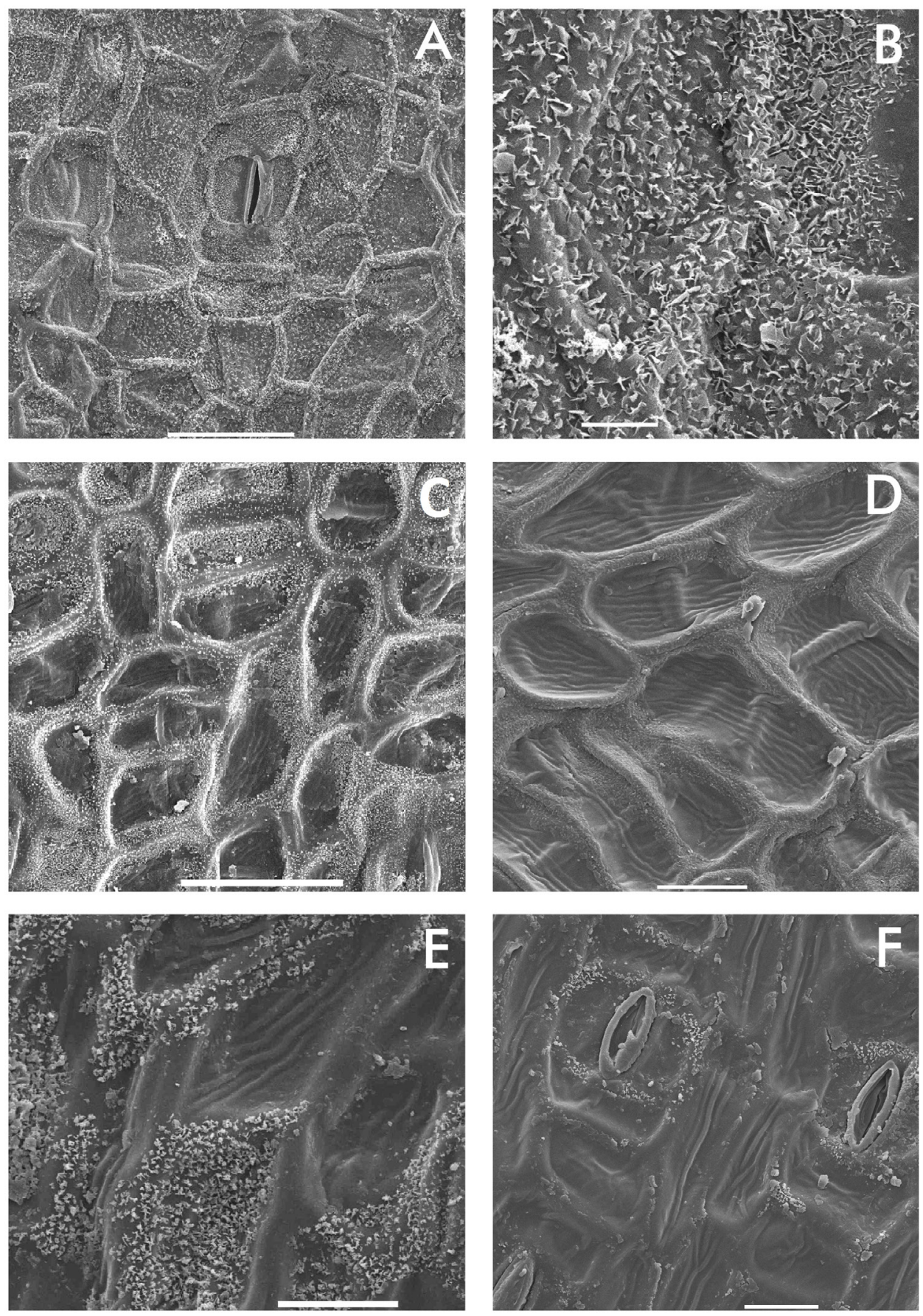

Fig. 1. Cera epicuticular y estrías epidérmicas en Cannaceae. A-B: C. glauca, cera en epifilo. B: C. glauca, detalle escamas de cera en hipofilo. C: C. fuchsina, cera en epifilo. D: C. coccinea, estrías y ceras en paredes de contacto celulares. E: C. ascendens, detalle escamas de cera en epifilo. F: C. ascendens, distribución de ceras y estrías en hipofilos. Barra: $A, C=50 \mu \mathrm{m}, B=5 \mu \mathrm{m}, \mathrm{D}-\mathrm{F}=20 \mu \mathrm{m}$. 
Bol. Soc. Argent. Bot. 52 (1) 2017
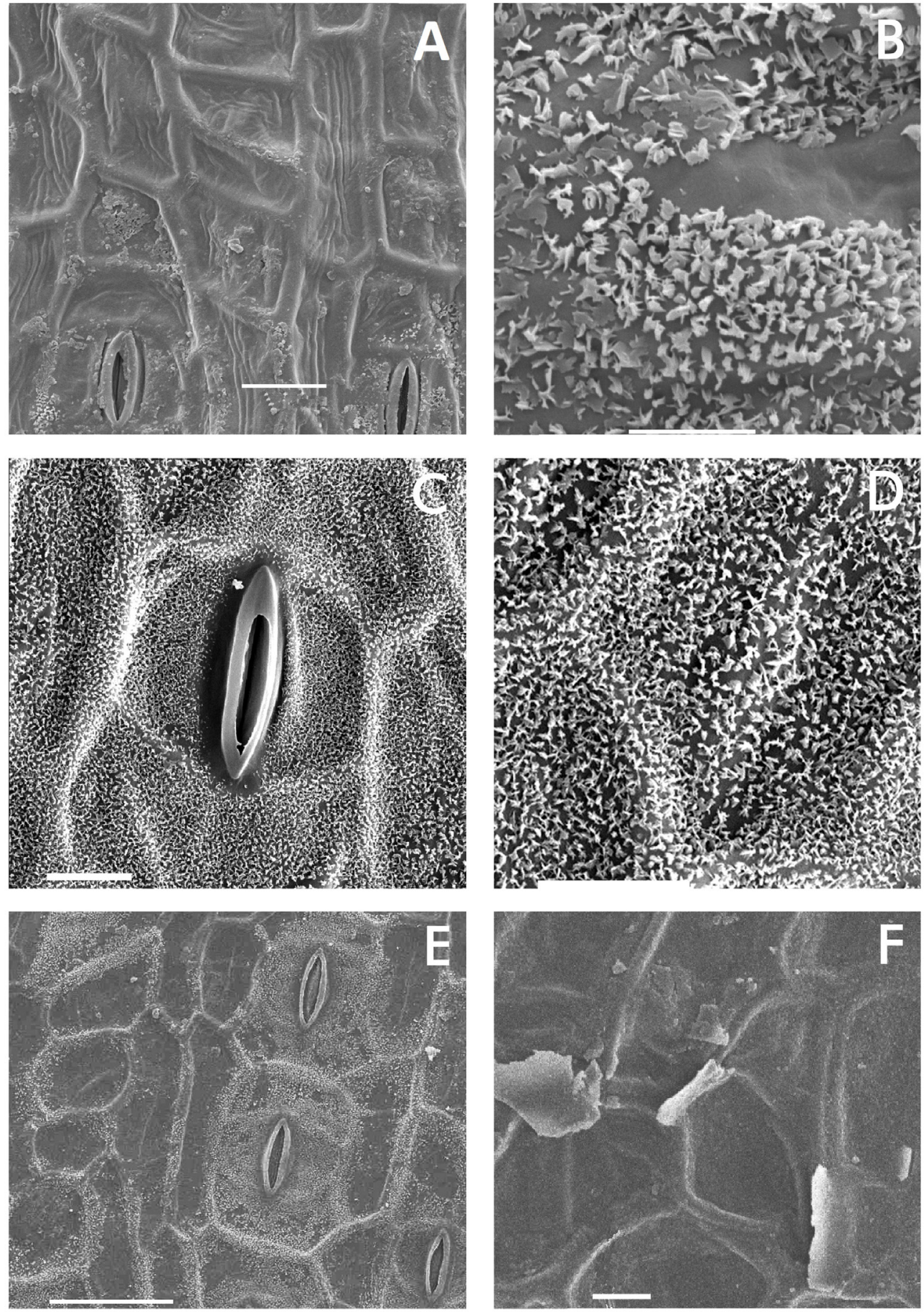

Fig. 2. Cera epicuticular y estrías epidérmicas en Cannaceae. A: C. variegatifolia, hipofilo. B: C. variegatifolia, detalle cera en epifilo. C: $C$. lineata, hipofilo. D: C. lineata, detalle escamas en hipofilo. E: $C$. tandilensis, cera en escamas en hipofilos. F: $C$. indica, cera en placas. Barra: $A, F=20 \mu \mathrm{m}, \mathrm{B}=5 \mu \mathrm{m}, \mathrm{C}-\mathrm{D}=$ $10 \mu \mathrm{m}, \mathrm{E}=50 \mu \mathrm{m}$. 
M. Ciciarelli et al. - Caracteres foliares Canna (Cannaceae)
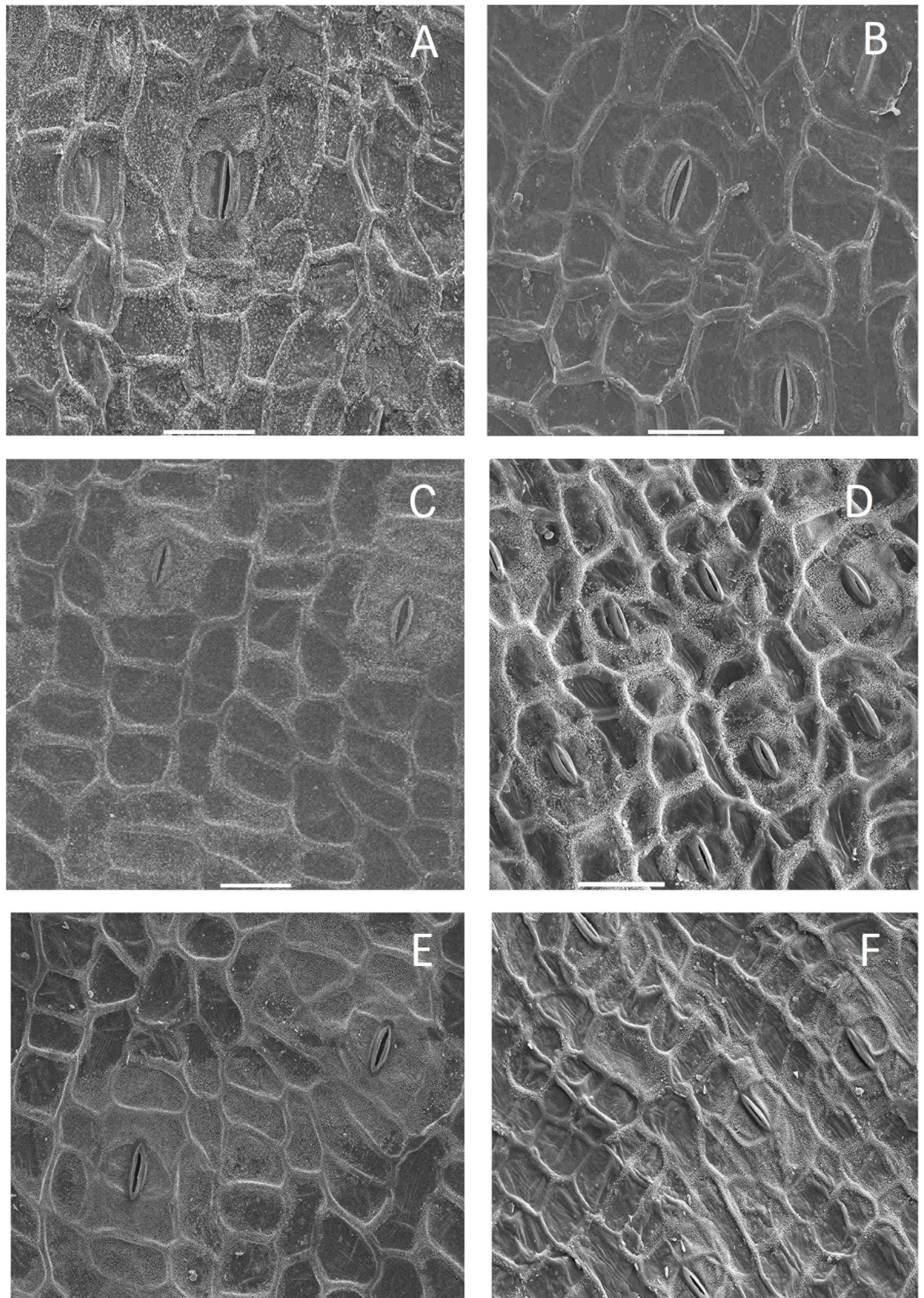

Fig. 3. Epidermis de especies de Canna: hileras sin estomas formadas por células isodiamétricas o hasta dos veces más largas que anchas. A: C. glauca. B: C. indica. C: C. tandilensis. D: C. fuchsina. E: C. tandilensis. F: C. lineata. A, C, E: epifilos. B, D, F: hipofilos. Barra: A-B, E-F=50 $\mu \mathrm{m}, \mathrm{C}-\mathrm{D}=20 \mu \mathrm{m}$. 
Bol. Soc. Argent. Bot. 52 (1) 2017
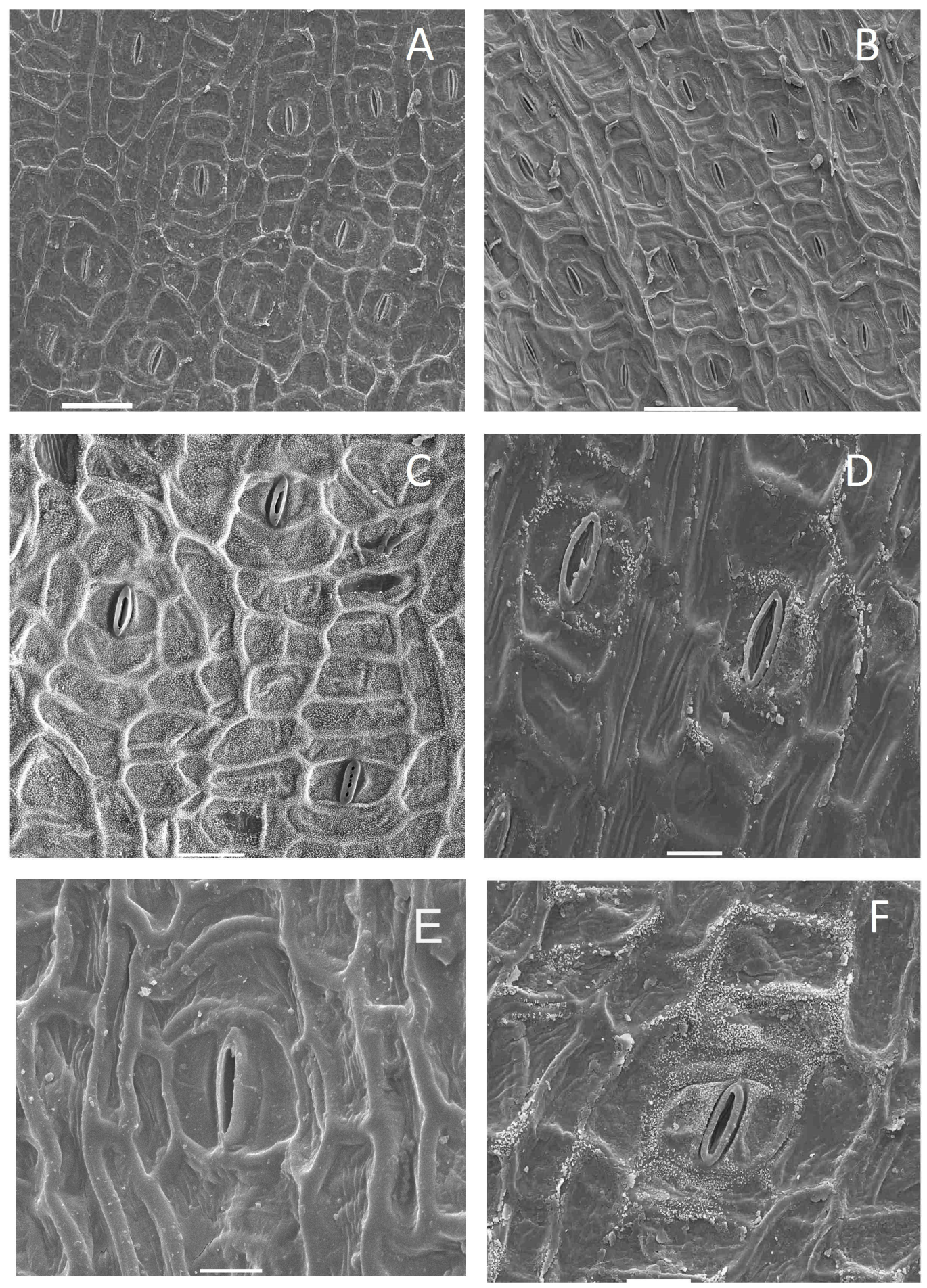

Fig. 4. Epidermis de especies de Canna: hileras sin estomas formadas por células hasta seis veces más largas que anchas. A: C. indica. B: C. coccinea. C: C. lineata. D: C. ascendens. E: C. paniculata. F: C. variegatifolia. A-E: hipofilos. F: epifilo. Barra: $A-B=100 \mu \mathrm{m}, C-D, F=20 \mu \mathrm{m}, E=50 \mu \mathrm{m}$. 
como en C. compacta, C. lineata y C. tandilensis y, ocasionalmente, en hipofilos de $C$. coccinea.

Las hileras que tienen estomas presentan células con sus contornos menos variables, raramente como polígonos alargados en sentido longitudinal sino más bien isodiamétricas o con relación 1/a de 2-4: 1 en sentido transversal, debido a su localización polar respecto de los estomas y contornos poligonales más o menos rectangulares, no angulosos, a veces casi oblongos curvos o semilunares, como en los hipofilos de C. coccinea (Fig. 4 B).

Estomas. Los estomas adultos tienen células oclusivas sobreelevadas, poros externos cutinizados, con un reborde engrosado de cutícula pura que puede ser liso o crenulado, poros internos simples lisos y células anexas en disposición paracítica. El reborde cuticular (poral) externo está presente en todas las especies; es más bien liso en los estomas de $C$. indica (Fig. 3 B), marcadamente engrosado en los estomas de C. variegatifolia (Fig. 5 D) y finamente crenulado a eroso en las demás especies (Fig. 5 A-F). Tienen dos células anexas en disposición típicamente paracítica, algo envolventes o totalmente convergentes en los polos de las oclusivas (Fig. 5 A-F). Estas células se originan a partir de la célula madre de las oclusivas, de manera mesógena, o bien son dos células protodérmicas que se adecuan a la disposición final paracítica en estomas desarrollados de manera perígena.

Ocasionalmente se observan disposiciones adultas tetracíticas, es decir, estomas paracíticos con un par adicional de anexas laterales en la hilera, como en $C$. coccinea y $C$. indica o bien hexacíticas, con un par de células de clara disposición polar, como en C. glauca. Las disposiciones tetracíticas son más frecuentes que las hexacíticas, éstas últimas son raras y probablemente deriven de una división extra de células polares que pertenecen a la hilera estomática que lleva estomas adultos y no a hileras contiguas que no tienen estomas. La actividad meristemática de las células iniciales (residuos protodérmicos) que darán origen a estomas no es tan claramente predecible si no se analiza la ontogenia.

Ontogenia de los estomas mesógenos de C. coccinea. El residuo meristemático protodérmico o inicial estomática experimenta dos o tres divisiones, en la última de las cuales se generan las células oclusivas (Fig. 6 A-E). Todas las células producidas en estas divisiones son hermanas ya que provienen del residuo meristemático protodérmico, que inicialmente tiene contorno aproximadamente cuadrangular y en su etapa más temprana aparece rodeado por cuatro células epidérmicas juveniles o protodérmicas (Fig. 6 A). La inicial origina dos células en una primera mitosis (Fig. 6 B). Una segunda división, por un plano paralelo al previo, da como resultado otras dos células y queda definido un conjunto de tres células contiguas en el que la central se comporta como la célula madre de las oclusivas, que se producen por medio de una tercera división (Fig. 6 D-F). Las anexas pueden dividirse nuevamente para formar un estoma adulto tetracítico, es decir, con dos anexas laterales a cada lado de las oclusivas (Fig. 6 F-G).

Ontogenia de estomas perígenos en C. glauca. La inicial protodérmica es cuadrangular y está rodeada por 4 células, también protodérmicas de disposición cruciforme, dos polares y dos laterales, todas con contorno poligonal y las laterales alargadas en sentido longitudinal (Fig. 7 A-B). El citoplasma de estas células es densamente granular y el núcleo, de gran tamaño en relación al tamaño celular general, presenta 1-2 nucléolos. Todo el grupo celular formado por el residuo protodérmico y las células epidérmicas juveniles adyacentes experimenta divisiones subsiguientes formando células derivadas en número variable (Fig. 7 C). Las más cercanas a la inicial o células vecinas se disponen como células anexas laterales y polares del estoma adulto. Antes de que se formen las oclusivas, se han observado las siguientes etapas: a) división de las células vecinas laterales para formar una célula de contacto, una a cada lado de la inicial (Fig. 7 C); b) formación de una célula de contacto polar inicialmente triangular mediante una sola división oblicua o recta de las células vecinas polares (estas divisiones en las células polares son poco frecuentes y predominan las mitosis en las laterales (Fig. 7 E); c) se define la cariocinesis de la inicial estomática, que se insinúa en la etapa (a), se forman las oclusivas y se observan dos o más células vecinas laterales (Fig. 7 D); la citocinesis de la inicial se completa cuando se ha formado una tercera célula lateral proveniente de la división de una de las células vecinas laterales; mientras que, en este estado una de las células polares suele encontrarse en división y tiene un tamaño notable en relación con la célula polar opuesta (Fig. 7 D-E). Cuando las células oclusivas están definidas se pueden observar dos células laterales a cada lado, la de contacto y la vecina que le dio origen y una división reciente en una célula vecina de posición polar. Estos fenómenos no siempre 
Bol. Soc. Argent. Bot. 52 (1) 2017
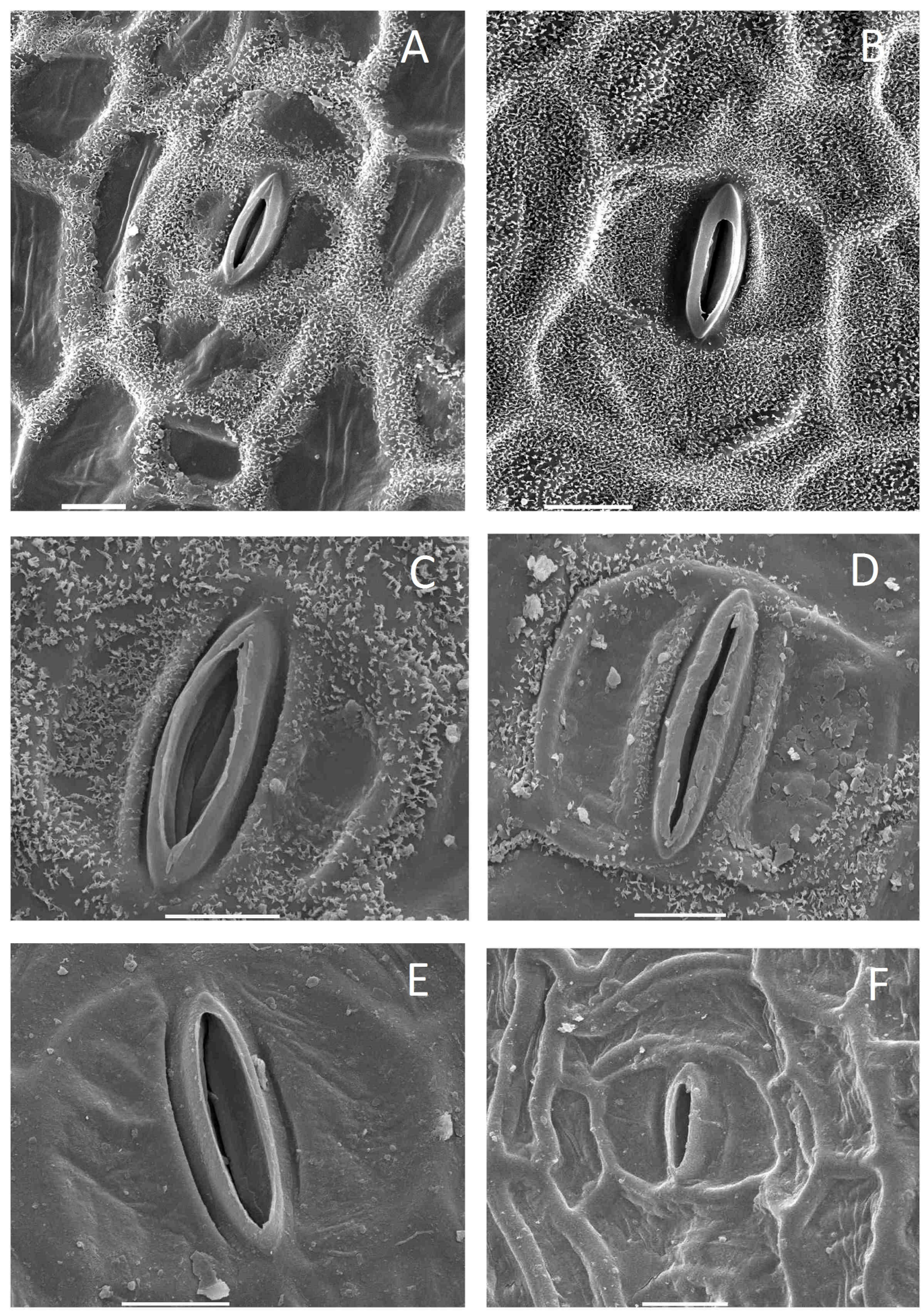

Fig. 5. Estomas adultos de especies de Canna. A: C. fuchsina. B: C. lineata. C: C. ascendens. D: C. variegatifolia. E: C. coccinea. F: C. paniculata. Barra: A-F=10 $\mu \mathrm{m}$. 
son sucesivos sino concomitantes o casi coincidentes en el tiempo.

En epidermis adultas hay hileras estomáticas con residuos protodérmicos no desarrollados que

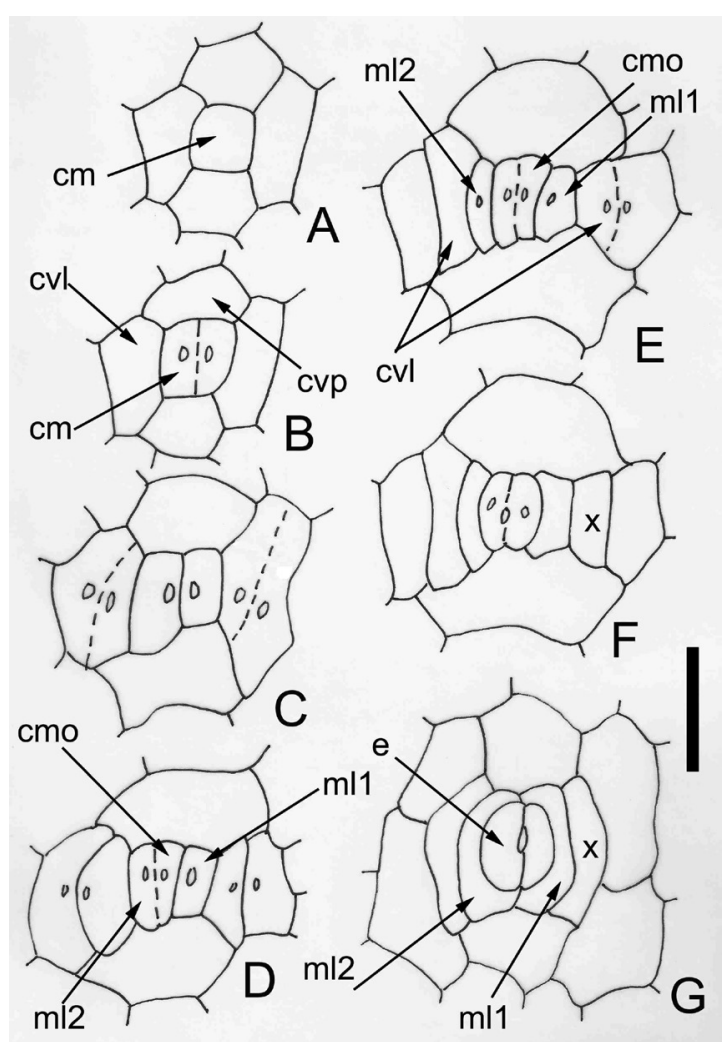

Fig. 6. Ontogenia de estomas en C. coccinea: desarrollo mesógeno. A: residuo meristemático protodérmico (inicial del estoma) rodeado por cuatro células en disposición tetracítica. B: primera división de la inicial. C: dos células meristemáticas derivadas de la inicial rodeadas de vecinas en división. D: segunda división del residuo meristemático, estado 3-celular y formación de dos anexas mesógenas laterales. E: tercera división del residuo meristemático, estado 4-celular, con dos anexas mesógenas y la célula madre de las oclusivas central en división. F: formación de dos oclusivas, anexas mesógenas laterales ya producidas. G: estoma adulto paracítico con dos anexas laterales mesógenas y otras dos, también en posición paracítica, originadas por la división de vecinas o de las mesógenas. $\mathrm{cm}$ = residuo meristemático protodérmico; $\mathrm{cmo}=$ célula madre de las oclusivas; $\mathrm{cvl}=$ célula vecina lateral; $\operatorname{cvp}=$ célula vecina polar; $\mathrm{e}=$ estoma adulto; $\mathrm{ml} 1$ y $\mathrm{ml} 2$ = anexas laterales mesógenas; $\mathrm{x}=$ anexas laterales mesógenas o perígenas. Barra: $35 \mu \mathrm{m}$. tienen contorno circular a poligonal, menor tamaño que las células adyacentes y están rodeados por 4-6 epidérmicas en disposición cruciforme o cíclica. El conjunto sugiere la célula inicial estomática y sus 4 células vecinas, pero es posible que no todas las iniciales estomáticas devengan finalmente en estomas funcionales.

Ocasionalmente, en todas las especies se presentan irregularidades en el patrón celular periestomático. Las células oclusivas pueden aparecer rodeadas por hasta 9 células dispuestas desordenadamente y con tamaños y contornos variados, con la presencia consecuente de un estoma formado por las
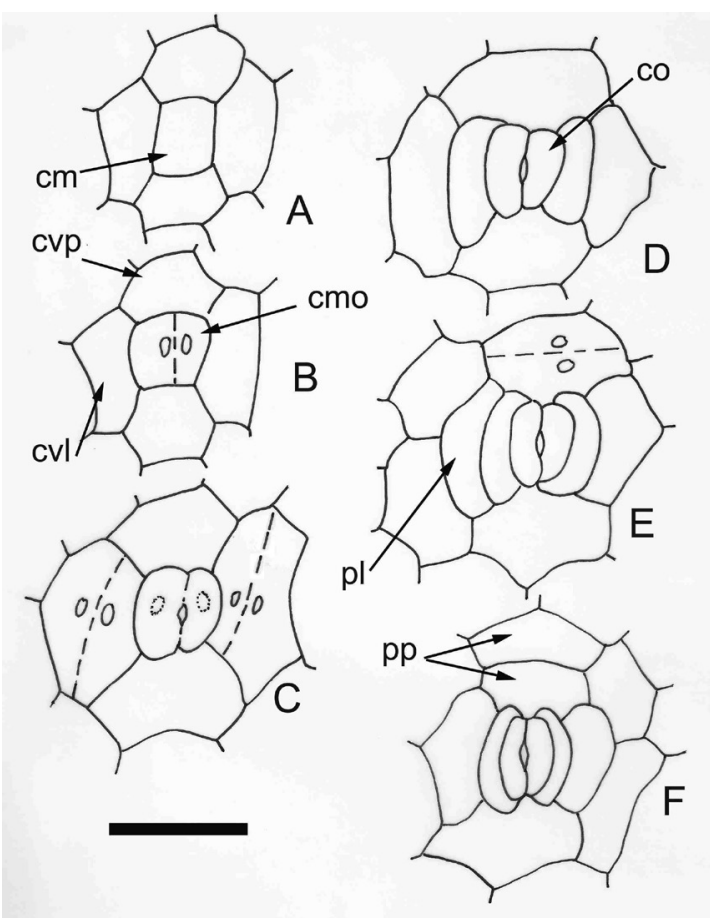

Fig. 7. Ontogenia de estomas en C. glauca: desarrollo perígeno. A: residuo meristemático protodérmico (inicial del estoma) rodeado por cuatro células en disposición tetracítica. B: primera división de la inicial. C: oclusivas juveniles, formación del poro y divisiones en las células periestomáticas vecinas. D: oclusivas adultas con dos vecinas curvadas en disposición paracítica. E-F: disposición paracítica adulta; eventual división en células vecinas polares. $\mathrm{cm}=$ residuo meristemático protodérmico; $\mathrm{cmo}$ = célula madre de las oclusivas; $\mathrm{co}=$ célula oclusiva; $\mathrm{cvl}=$ célula vecina lateral; $\mathrm{cvp}=$ célula vecina polar; $\mathrm{pl}=$ anexas laterales perígenas; $\mathrm{pp}=$ anexas polares perígenas. Barra: $35 \mu \mathrm{m}$. 
oclusivas y un número variable de células vecinas morfológicamente diferentes, perdiéndose de vista la disposición tetracelular corriente. En C. glauca se han observado estomas acíticos, es decir, sin células anexas y hemiparacíticos, es decir con una sola anexa. En general, en todas las especies se encuentra alguna clase de estoma atípico, que puede relacionarse con agrandamiento notable de las dos anexas laterales, divisiones adicionales en planos variables de una o ambas anexas laterales, presencia de anexas de diferentes tamaños, estomas contiguos que comparten anexas o, incluso, oclusivas, etc. Estas desviaciones de la disposición típica se localizan especialmente en áreas apicales adultas, donde se encuentran las zonas de actividad meristemática foliar más intensa.

Los caracteres más destacables de la morfología foliar de las especies de Canna se incluyen en la Tabla 1.

Tabla 1. Caracteres foliares en Cannaceae.

\begin{tabular}{|c|c|c|c|c|c|c|c|c|}
\hline $\begin{array}{l}\text { Ecología de } \\
\text { las especies }\end{array}$ & Especies & $\begin{array}{l}\text { Contorno } \\
\text { foliar } \\
\text { y relación I/a }\end{array}$ & $\begin{array}{l}\text { Espesor } \\
\text { de la } \\
\text { pared en } \\
\text { epifilos } \\
(\mu \mathrm{m})\end{array}$ & $\begin{array}{l}\text { Espesor de } \\
\text { la pared en } \\
\text { hipofilos } \\
\text { en }(\mu \mathrm{m})\end{array}$ & $\begin{array}{l}\text { Dimensiones } \\
\text { estomas } \\
\text { epifilos }(\mu \mathrm{m})\end{array}$ & $\begin{array}{l}\text { Dimensiones } \\
\text { estomas } \\
\text { hipofilos }(\mu \mathrm{m})\end{array}$ & $\begin{array}{l}\text { Densidad } \\
\text { estomas } \\
\text { epifilos }\left(^{*}\right)\end{array}$ & $\begin{array}{l}\text { Densidad } \\
\text { estomas } \\
\text { hipofilos }\left(^{*}\right)\end{array}$ \\
\hline \multirow[t]{2}{*}{ Anfibia } & C. glauca & $\begin{array}{c}\text { linear a } \\
\text { linear } \\
\text { lanceolado } \\
(6: 1)\end{array}$ & 6 & $8-10$ & $31 \times 23$ & $33 \times 25$ & (6) 10 (17) & (17) 26 (32) \\
\hline & C. fuchsina & $\begin{array}{c}\text { lanceolado } \\
\text { angosto } \\
(3: 1)\end{array}$ & 4 & 6 & $36 \times 22$ & $38 \times 26$ & (4) 9 (10) & (29) 31 (34) \\
\hline $\begin{array}{l}\text { Terrestre, suelos } \\
\text { húmedos e } \\
\text { inundables, } \\
\text { ribereñas, } \\
\text { humedales }\end{array}$ & C. ascendens & $\begin{array}{l}\text { elíptico } \\
(3-4: 1)\end{array}$ & 6 & 8 & $29 \times 19$ & $30 \times 19$ & (2) 7 (11) & (17) 32 (51) \\
\hline $\begin{array}{l}\text { Terrestre, suelos } \\
\text { húmedos e } \\
\text { inundables, } \\
\text { ribereñas, } \\
\text { humedales }\end{array}$ & C. lineata & $\begin{array}{l}\text { lanceolado } \\
\text { angosto } \\
(5-4: 1)\end{array}$ & 5 & 5,5 & $31 \times 20$ & $37 \times 22$ & (11) 13 (18) & (27) 31 (36) \\
\hline $\begin{array}{l}\text { Terrestre, suelos } \\
\text { húmedos e } \\
\text { inundables, } \\
\text { ribereñas, } \\
\text { humedales }\end{array}$ & C. tandilensis & $\begin{array}{l}\text { lanceolado } \\
\text { angosto } \\
(5-4: 1)\end{array}$ & 5 & 4 & $28 \times 12$ & $28 \times 16$ & (6) 8 (10) & (32) 39 (43) \\
\hline $\begin{array}{l}\text { Terrestre, suelos } \\
\text { húmedos e } \\
\text { inundables, } \\
\text { ribereñas, } \\
\text { humedales }\end{array}$ & $\begin{array}{c}\text { C. } \\
\text { variegatifolia }\end{array}$ & $\begin{array}{c}\text { lanceolado } \\
(3-4: 1)\end{array}$ & 5,3 & 8 & $30 \times 23$ & $32 \times 24$ & (4) $5(6)$ & (26) 28 (29) \\
\hline $\begin{array}{l}\text { Terrestre, selvas } \\
\text { Iluviosas }\end{array}$ & C. compacta & $\begin{array}{c}\text { elíptico a } \\
\text { oblongo } \\
(2-3: 1)\end{array}$ & 5 & 6 & $27 \times 20$ & $30 \times 22$ & (0) 6 (13) & (18) 27 (34) \\
\hline $\begin{array}{l}\text { Terrestre, selvas } \\
\text { Iluviosas }\end{array}$ & C. paniculata & $\begin{array}{c}\text { lanceolado } \\
(3-4: 1)\end{array}$ & 3 & 3,4 & $22 \times 17$ & $22 \times 16$ & (0) 5 (11) & (41) 51 (70) \\
\hline $\begin{array}{l}\text { Terrestre,suelos } \\
\text { húmedos } \\
\text { removidos, } \\
\text { humedales, a } \\
\text { veces ruderales }\end{array}$ & C. coccinea & oblongo( $2: 1)$ & 7 & 8 & $26 \times 19$ & $28 \times 23$ & (4) 5 (6) & (23) 31 (41) \\
\hline $\begin{array}{l}\text { Terrestre,suelos } \\
\text { húmedos } \\
\text { removidos, } \\
\text { humedales, a } \\
\text { veces ruderales }\end{array}$ & C. indica & $\begin{array}{l}\text { elíptico } \\
(2-3: 1)\end{array}$ & 4 & 8 & $32 \times 25$ & $34 \times 28$ & (2) 5 (6) & (16) 19 (22) \\
\hline
\end{tabular}




\section{Discusión}

Los caracteres vegetativos estudiados en especies de Canna fueron los siguientes: presencia y tipos de indumento, contorno foliar, tipos y distribución de ceras epicuticulares, modelos epidérmicos, ordenamiento celular de epifilos e hipofilos, espesor de la pared externa de las células epidérmicas, contorno del estoma paracítico, tipos, densidad y dimensiones de los estomas adultos. Este conjunto de rasgos se analizó en relación con el hábitat natural de las plantas.

La ontogenia se ilustró en dos especies, $C$. glauca y C. coccinea. El tipo de desarrollo más frecuente es el perígeno, pero se encontró un modelo de desarrollo mesógeno en estomas de $C$. coccinea, que se menciona por primera vez para la familia.

El indumento es raro en Canna; aquí se observó en dos especies, C. compacta, con pelos unicelulares y $C$. paniculata, con pelos diversos y escamas.

Los caracteres analizados tienen valor diagnóstico variable. La combinación del contorno foliar, la presencia y tipos de ceras y ornamentaciones cuticulares, la densidad y tamaño de los estomas adultos y la presencia de indumento pueden ser útiles en el nivel específico. Los contornos foliares se sugieren como caracteres diagnósticos específicos, aunque también puede decirse que ciertos contornos parecen predominar en grupos de especies y en relación con su hábitat: en las especies anfibias predominan las hojas lineares erguidas, en las anfibias facultativas y riparias las hojas lanceoladas patentes o adpresas con el tallo y en los elementos higrófitos terrestres son característicos los tipos latifoliados, reflejos, con contornos ovados, oblongos o elípticos, es decir, que podría haber una relación entre ecología y desarrollo de la lámina, además del valor diagnóstico específico del rasgo en cuestión.

Los modelos epidérmicos, generalmente poligonales o subpoligonales, con contornos celulares en forma de polígonos más bien redondeados a levemente sinuosos y no rectos en higrófitos de selva, como C. paniculata, parecen, por el momento, característicos de grupos de especies, y no claramente específicos. Los diámetros celulares predominantes de las hileras que no incluyen estomas determinan dos grandes grupos: las que presentan células isodiamétricas dos veces más largas que anchas y las que están formadas por células alargadas, hasta seis veces más largas que anchas. Estos dos tipos de hileras pueden estar presentes en epifilos o hipofilos de la misma especie, como es el caso de $C$. coccinea y $C$. indica. Sin embargo, la combinación del tipo de hileras sin estomas, con los tipos de estomas adultos, las ceras y las estrías sí constituye un conjunto que funciona como diagnóstico en el nivel específico.

Algunos caracteres foliares muestran una relación con el hábitat que debe destacarse.

Las especies anfibias, como C. glauca y C. fuchsina, tienen cera abundante en escamas densas, cutículas estriadas y modelos epidérmicos poligonales con las paredes rectas que se encuentran entre las más engrosadas de las especies estudiadas, como es el caso de C. glauca.

Las especies riparias, terrestres, de pastizales húmedos o inundables y bordes de corrientes de agua, ampliamente tolerantes a la radiación solar, como C. ascendens, C. lineata, C. tandilensis y C. variegatifolia presentan cera en laminillas o escamas densas pero comparativamente muy pequeñas y localizadas en áreas determinadas discontinuas, la cutícula tiene estrías poco marcadas o carece de ellas y los modelos epidérmicos poligonales presentan células con paredes de espesor considerable, algunos similares a los observados en especies anfibias.

Los higrófitos terrestres de áreas selváticas y suelos húmedos como $C$. coccinea, $C$. compacta y $C$. indica tienen cera escasa y dispersa, en general sobre las paredes de contacto de las células epidérmicas o bien en placas delgadas que se desprenden; las cutículas tienen estriaciones leves o ausentes y las paredes de la células epidérmicas pueden estar comparativamente poco engrosadas, aunque la excepción a esta generalización es $C$. coccinea la especie con los mayores espesores parietales del grupo. Canna compacta, un higrófito de selvas lluviosas se distingue por el indumento lanuginoso formado por pelos unicelulares, mientras que C. paniculata, otro higrófito de selvas lluviosas, se caracteriza por la presencia de indumento piloso lanuginoso morfológicamente más diverso y escamas; la epidermis presenta papilas, modelos subsinuosos, la mayor densidad estomática y las paredes epidérmicas más delgadas de todas las especies analizadas. 
La cera abundante y las estrías epidérmicas se han señalado como respuestas fisiológicas tanto en plantas de zonas áridas como en plantas anfibias y de selvas lluviosas, con diferentes funciones pero siempre relacionadas con el control de la pérdida de agua o el exceso de ésta en la superficie foliar. En plantas anfibias o ribereñas el exceso de pérdida de agua puede acentuarse en momentos en que cede la inundación, por la exposición o por ambas causas, y la presencia de ceras también se ha analizado en relación con la refracción de la luz en plantas expuestas a la radiación solar o en relación con la capacidad de esas ceras de fundirse con la temperatura y actuar como capa protectora renovable. En hidrófitos de selva es necesario eliminar rápidamente al agua de las superficies foliares y las ceras permiten una superficie deslizante para esa función. El balance de las funciones, por lo tanto, estaría relacionado no sólo con la presencia y distribución de estas ceras sino con su composición. Aquí no se ha estudiado la composición química de las ceras presentes, pero tanto en los higrófitos de selvas como en los elementos anfibios y ribereños la composición de las ceras presentes sería una explicación plausible aunque no la única (Kerstiens, 1996; Barthlott \& Neinhuis 1997; Krauss et al., 1997; Riederer \& Schreiber, 2001; Goodwin \& Jenks, 2005; Holloway \& Jeffree, 2005; Jetter et al., 2006; Buschhaus \& Jetter, 2011, entre otros).

Observaciones efectuadas en otras Zingiberales que crecen en un área y hábitats similares ecológicos sugieren que tanto las ceras epicuticulares como el ordenamiento celular (modelos epidérmicos) podrían ser más comunes en el orden y no exclusivos de Cannaceae (Arrieche \& Sanabria, 1995; Brilhante de Albuquerque \& de Jesús Neves, 2004; Martins et al. 2010; Sumardi \&Wulandari, 2010; Torres Boeger et al. 2014, y datos no publicados de las autoras), y es posible que los tipos de epidermis descritos puedan considerarse rasgos de familia, como ocurriría con las ceras, ya que la presencia de cera en escamas está difundida en el orden pero el tamaño, distribución y localización de las escamas o placas varían en las familias del orden (datos no publicados). Por lo conocido hasta el momento, es posible sugerir que el patrón epidérmico podría ser un carácter más en común, compartido por las familias del orden Zingiberales, con modelos de organización similares, especialmente Cannaceae, Heliconiaceae, Marantaceae, Musaceae y Zingiberaceae (Arrieche \& Sanabria, 1995; Kress et al., 2001).

Los estomas paracíticos presentes en Cannaceae también se mencionan para otras Zingiberales, como Alpinia zerumbet (Pers.) Burtt. \& Smith (Brilhante de Albuquerque \& de Jesus Neves, 2004), Thalia geniculata L., algunas especies de Strelitzia Aiton y Musa L. (Stebbins \& Khush, 1961; Sumardi \& Wulandari, 2010) y en varias especies de Heliconia L. (Arrieche \& Sanabria, 1995), en las que se describen también las hileras celulares con y sin estomas, todos con anexas en disposición paracítica. Los estomas de Hedychium J. König se describen como tetracíticos (Torres Boeger et al., 2007). En todos los trabajos mencionados los tamaños estomáticos tienen valores similares a los encontrados en Cannaceae, con la mayor longitud en 28-38 (40) $\mu \mathrm{m}$ y una anchura entre 24-28 (32) $\mu \mathrm{m}$, de modo que las dimensiones estomáticas podrían ser un rasgo compartido por varias familias del orden, no típico de Cannaceae.

\section{Agradecimientos}

Este trabajo fue realizado en el marco del Programa de Incentivos para Docentes Investigadores de la Universidad Nacional de La Plata (Argentina).

\section{Biblografia}

ARRIECHE, D. \& M. E. SANABRIA. 1995. Ultraestructura de la epidermis foliar de cinco especies de Heliconia. Bioagro 7: 63-69.

BAKER, E. A. 1982. Chemistry and morphology of plant epicuticular waxes: In: CUTLER, D. J., K.L. ALVIN \& C. E. PRICE (eds.), The Plant Cuticle, pp. 139165. Academic Press, London.

BARANOVA, M. 1992. Principles of comparative stomatographics studies of flowering plants. Bot. Rev. 58: 49-99.

BARTHLOTT, W., C. NEINHUIS, D. CUTLER, F. DITSCH, I. MEUSEL, I. THEISEN \& $\mathrm{H}$. WILHELMI. 1998. Classification and terminology of plant cuticular waxes. Bot. J. Linn. Soc. 126: 237260.

BIANCHI, G. 1995. Plant waxes. In: HAMILTON, R. J. (ed.), Waxes: Chemistry, Molecular Biology and Functions, pp 175-222. The Oily Press, Dundee. 


\section{Ciciarelli et al. - Caracteres foliares Canna (Cannaceae)}

BRILHANTE DE ALBUQUERQUE, E. S. \& L. DE JESUS NEVES. 2004. Anatômica foliar de Alpinia zerumbet (Pers.) Burtt\& Smith (Zingiberaceae). Acta Bot. Bras 18: 109-121.

BUSCHHAUS, C. \& R. JETTER. 2011. Composition differences between epicuticular and intracuticular wax substructures: how do plants seal their epidermal surfaces. J. Exp. Bot. 62: 841-853.

CICIARELLI, M. M. 2007. Canna ascendens Ciciarelli (Cannaceae) una nueva especie de la provincia de Buenos Aires y comentarios de otras especies argentinas de este género. Darwiniana 45: 188-200.

CICIARELLI, M. M. 2014. Canna lineata Ciciarelli, una nueva especie para los humedales bonaerenses, Argentina. Bot. Complut. 38: 115-121.

CICIARELLI, M. M. 2015. Canna tandilensis Ciciar. (Cannaceae-Zingiberales), una especie nueva para Argentina. Bot. Complut. 39: 87-96.

CICIARELLI M. M. \& C. H. ROLLERI. 2008. Morfología, taxonomía y caracterización de siete especies neotropicales del género Canna (Cannaceae). Bot. Complut. 32: 157-184.

CICIARELli, M. M., C. H. ROLLERI, C. H. \& GONZALEZ DUBOX, M. C. 2010. Canna fuchsina, una especie nueva para la ciencia, sus relaciones con otras especies silvestres del género y con el grupo $C$. $x$ generalis (Cannaceae-Zingiberales). Bot. Complut. 34: 49-55.

CROXDALE, J. 1998. Stomatal patterning in monocotyledons: Tradescantia as a model system. $J$. Exp. Bot. 49: 279-292.

EGLINTON, G., R.J. HAMILTON, R.A. RAPHAEL, \& A.G. GONZALEZ. 1962. Hydrocarbon constituents of the wax coatings of plant leaves: a taxonomic survey. Nature 193: 739-42.

FALKENBERG, P., 1876. Vergleichende Untersuchungen uber den Bau der Vegetations organe der Monokotiledonen. Stuttgart.

FOSTER, A.S., 1949. Practical Plant Anatomy. Van Nostrand Book Co., Princeton.

GOODWIN, S. M. \& M.A. JENKS. 2005. Plant cuticle function as a barrier to water loss. In: JENKS, M. A. \& P. M. Hasagawa (eds.), Plant Abiotic Stress, pp. 14-36. Blackwell, Oxford.

GURR, L. 1966. The Rational Use of Dyes in Biology. Williamson and Wilkers, Baltimore.

HOLLOWAY, P. J. \& C. E. JEFFREE. 2005. Epicuticular waxes. In: THOMAS, B., D. J. MURPHY \& B. G. MURRAY (eds.), Encyclopedia of Applied Plant Sciences vol. 3, pp. 1190-1204. Academic Press, Oxford.

JEFFREE, C. E. 2006. The fine structure of the Plant Cuticle In: RIEDERER, M \& C. MÜLLER (eds.), Biology of the Plant Cuticle, pp. 11-25. Blackwell Publishing, Oxford.
JETTER R, L. KUNST \& A. L. SAMUELS. 2006. Composition of plant cuticular waxes. In: RIEDERER, M. \& C. MÜLLER, (ed.), Biology of the Plant Cuticle, pp. 145-181. Blackwell Publishing, Oxford.

JOHANSEN, D. A. 1940. Plant Microtechnique. McGraw-Hill, New York.

KERSTIENS, G. 1996. Cuticular water permeability and its physiological significance. J. Exp. Bot. 47: 1813-1832

KRAUSS, P., C. MARKSTÄDTER \& M. RIEDERER. 1997. Attenuation of UV radiation by plant cuticles from woody species. Plant Cell Environ. 20: $1079-1085$

KRESS, W. J., L. M. PRINCE, W. J. HAHN \& E. A. ZIMMER. 2001. Unraveling the Evolutionary radiation of the families of the Zingiberales using morphological and molecular evidence. Syst. Biol. 50: 926-944.

LEBLOIS, A. 1887. Recherches sur l'origine et le developpement des canaux sécréteurs et des poches sécrétrices. Ann. Sci. Nat. Bot. sér.7: 247-330.

LOV, L. 1926. Zur Kenntnis der Entfaltunszellen monokotyler Blater. Flora. (Jena) 120: 283-343.

LUTZ, L. 1897. Gomme de Canna. Bull. Soc. Bot. France. 44: 48-51.

MARTINS, M. B. G, A. L. C. CARAVANTE, B. APPEZZATO-DA-GLÓRIA, M. K. M. SOARES, R. R. D. MOREIRA \& L. E. SANTOS. 2010. Caracterização anatômica e fitoquímica de folhas e rizomas de Hedychium coronarium J. König (Zingiberaceae). Rev. Brás. Plantas Med. 12: 179-187.

MEUSEL, I. , E. LEISTNER \& W. BARTHLOTT. 1994. Chemistry and micromorphology of compound cuticular wax crystalloids (Strelitzia type). Plant Syst. Evol. 193:115-123.

NEINHUIS, C. \& W. BARTHLOTT. 1997. Characterization and distribution of waterrepellent, self-cleaning plant surfaces. Ann. Bot. 79: $667-677$.

OLATUNJI, O. A., 1980. The structure and development of stomata in some Zingiberales. Notes Roy. Bot. Gard. Edinburgh 38: 499-515.

PANT, D. D. 1965. Onthogeny of stomata and other homologous structures. Plant Sci. Ser. (Allahabad) 1: 1-24.

PASSARELli, L. M., C. H. ROLlERI, M. M. CICIARELLI, A. C. DEDOMENICI \& G. E. GONZÁLEZ. 2014. Flora vascular de humedales permanentes y transitorios bonaerenses (Buenos Aires, Argentina). Bot. Complut. 38: 137-152.

PAYNE, W. W. 1979. Stomatal patterns in embryophytes: their evolution, ontogeny and interpretation. Taxon 28: 117-32. 
RASMUSSEN, H. 1981. Terminology and classification of stomata - a critical survey. Bot. J. Linn. Soc. 83: 199-212.

RIEDERER, M. \& L. SCHREIBER. 2001. Protecting against water loss: analysis of the barrier properties of plant cuticles. J. Exp. Bot. 52: $2023-$ 2032.

SOLEREDER, H. \& F. MEYER. 1930. Systematiche anatomie der Monokotyledonen. Verlag von Gebruder Borntraeger, Berlin.

STEBBINS, G. L. \& G. S. KHUSH. 1961.Variation in the organization of the stomatal complex in the leaf epidermis of monocotyledons and its bearing on their phylogeny. Am. J. Bot. 48: 51-59.

SUMARDI, I. \& M. WULANDARI. 2010. Anatomy and morphology character of five Indonesian banana cultivars (Musa spp.) of different ploidy level. Biodiversitas 11: 167-175.

STRASBURGER, E. 1866. Ein Beitrage zur Entwicklungsge- schichteder Spaltoffnungen. Jahrbücher für Wissenschaftliche Botanik. Jahrb. Wiss. Bot. 5: 297- 342.

THEOBALD, W. L., J. L. KRAHULIK \& R. C. ROLLINS. 1979. Trichome description and classification: In: METCALFE, C. \& L. CHALK (eds.). Anatomy of the Dycotiledons, pp. 40-53. Oxford University Press, Oxford.

TOGNINI, F. 1897. Contribuzione allo studio della organo- genia comparata degli stomi. Atti. Ist. Bot. Univ. Pavia 4: 1-42.

TOMLINSON, P. B. 1961. The anatomy of Canna. J. Linn. Soc. (Bot.) 56: 467-473.
TOMLINSON, P. B. 1962. Phylogeny of the Scitaminae. Morphological and anatomical considerations. Evolution 16: 192-213.

TOMLINSON, P. B., 1969. Commelinales-Zingiberales. In: METCALF, C. R. (ed.). Anatomy of the Monocotyledons 3, pp. 365-373. Oxford University Press, Oxford.

TOMLINSON, P. B., 1974. Development of the stomatal complex as a taxonomic character. Taxon 23: 109128.

TORRES BOEGER, M. R., M. W. BARBOSA DE OLIVEIRA PIL, \& N. B. FILH 2007. Arquitetura foliar comparativa de Hedychium coronarium J. Koenig (Zingiberaceae) y de Typha domingensis Pers. (Typhaceae). Iheringia 62: 113-120.

VESQUE, M. J. 1889. De I'emploi des caracteres anatomiques dans la classification des Vegetaux. Bul. Soc. Bot. France 36: 41-77.

VON WETTSTEIN- KNOWLES, P. 1995. Biosynthesis and genetics of waxes. In: HAMILTON, R.J. (ed.). Waxes: Chemistry, Molecular Biology and Functions, pp. 131-174. The Oily Press, Dundee.

WILKINSON, H. P. 1979. The plant surface (mainly leaf). In: METCALF, C. R. \& L. CHALK (eds.), Anatomy of the Dycotiledons 1, pp. 97-165. Clarendon Press, Oxford.

Recibido el 12 de julio de 2016, aceptado el 11 de octubre de 2016. 
M. Ciciarelli et al. - Caracteres foliares Canna (Cannaceae)

Apéndice 1. Ejemplares de referencia seleccionados.

\section{Cannaceae}

Canna ascendens Ciciar. Darw.45:188-200.2007. Tipo: ARGENTINA. Prov. Buenos Aires: Dpto. La Plata, Camino Centenario y Lacroze, Ciciarelli 5 (Holotypus LP). Paratipos: ARGENTINA. Prov. Buenos Aires: Dpto. San Pedro, Panamé, Ciciarelli 17 (LP); Dpto. Leandro N. Alem Vedia, Vedia, sobre ruta 7, Ciciarelli, 6 (LP); Dpto. La Plata, Ringuelet, Camino Centenario y 519, Ciciarelli 7 (LP); City Bell, Camino Centenario y Lacroze, Ciciarelli 8 (LP).

Canna coccinea Mill. ARGENTINA: Prov.Tucumán: Dpto. Capital, Empalme Centenario, Colmenal, Villa 131568 (LIL); entre Capital y Muñecas, Meyer 13592 (LIL); Muñecas, Schreiber 51603 (LIL); Dpto. Tafi del Valle, Venturi 222 (SI); a orillas de la vía de Tafí al Cadillal, Herrera 345 (LIL); Prov. Misiones: Dpto. Candelaria, Santa Ana, Rodríguez 672 (SI); Dpto. General M. Belgrano, Bernardo de Irigoyen, Zardini, Deginiani 901 (SI); Prov. Corrientes: Dpto. Capital, Avda. Libertad, acceso a la ciudad de Corrientes Guaglianone et al. 15871 (NY); Formosa, Dpto. Monte Lasten, Jorgensen 2303 (LIL); Prov. Chaco: Schulz \& Benítez 26 (LP); Prov. Córdoba, Dpto. Colón, Quebrada de Ascochinga, Giardelli 1194 (SI); Prov. Buenos Aires: Dpto. Ensenada, Punta Lara, Fabris 7517 (LP); Cabrera 4902 (LP); Río Santiago s/c 26942 (SI); Dpto. Tigre, Tigre, Hicken 26940 (SI); Paraná de las Palmas, Burkart 9260 (SI); Dpto. La Plata, City Bell, Ciciarelli 1 (LP); Dpto. Capital, Palermo, Hicken 26941 (SI).

Canna compacta Rosc. ARGENTINA. Prov. Jujuy: Dpto. Dr. Manuel Belgrano, Yala, Fabris 8547 (LP). Yala, Cabrera y Fabris 20005 (LP). Quebrada de Yala, Cabrera y Fabris 19964 (LP). Camino de Reyes a Yala, Cabrera y Kiesling 20107 (LP). Dpto Santa Bárbara, Sierra de Santa Bárbara, El Tipal, Fabris 8087 (LP). Prov. Salta: Dpto Capital, ruta 51 entre km 1578 y 1579, Koyama et al. 15860, 15861,15862, (NY, SI), Dpto. Orán, San Ramón de la Nueva Orán, Finca de Yakulikas, al SE de las serranías Las Pavas cerca de la localidad Aguas Blancas, Palací 3 (LP). Dpto. Anta, Parque Nacional El Rey, Chalukian 7 (LP). Dpto. Rosario de Lerma, Santa Rosa de Tastil, Fabris 8548 y 8549 (LP). Dpto. Metán, cerca de la Represa Blanca Sierra, Herrera 101 (LIL). Prov.Tucumán: Dpto. Chicligasta, Puesto Las Pavas, Pueblo Viejo a Casa de Piedra, Legname \& Cuezzo 5561 (LIL). Río Chico, Escaba, Monetti 1591 (LIL). Dpto. Tafi Viejo, entre Tafí Viejo y Los Nogales, ruta 9, Meyer \& Vaca Gomez 22313 (LIL). Dpto. Famaillá, Río Colorado, Terrible 323 (LIL). Prov. San Luis: Dpto. Coronel Pringles, El Trapiche, Ciciarelli 22 (LP). BRASIL. Prov. Amazonas, Itapiranga, Smith \& Reitz 12640 (SI).

Canna fuchsina Ciciar. Bot. Complutensis 34: 49-55. 2010. PARATIPOS. ARGENTINA. Prov. Buenos Aires: Dpto. La Plata, City Bell, Pellegrini e/ 5 y 6. Ciciarelli 18 (LP). Prov. Santa Fe: Dpto. Las Colonias, Providencia, al borde de caminos, Ciciarelli 15 (LP).

Canna glauca L. ARGENTINA. Prov. Jujuy: Dpto. Santa Bárbara, ruta 1, Morrone 3465 (SI). Dpto. San Pedro, entre San Pedro y Chalicán, Cabrera et al. 34691 (SI). Prov. Tucumán: Dpto. Capital, Río Lules a San Felipe, Schreiter 780 (SI). Prov. Salta: Dpto Orán, San Ramón de la Nueva Orán, entre Río Piedras y Urundel, Vervoorst et al. 4649 (SI). Prov. Formosa: Dpto. Laishi, Ruta prov. 5 entre Laishi y Tatané, Guaglianone et al. 716 (SI). Prov. Chaco: Dpto. $1^{\circ}$ de Mayo, ruta 11, $7 \mathrm{~km}$ al N de María Belén, Guaglianone et al 15867, 15870 (SI). Ruta 11, 12 km de María Belén, Guaglianone et al. 15867 (SI). Prov. Corrientes: Dpto. Saladas, Tabay, s/c 3692 (SI). Prov. Entre Ríos: Dpto. Concordia, Concordia, Schulz 134605 (LIL); Dpto. Federación, Isthilart, Schulz 559 (SI). Prov. Buenos Aires: Dpto. Ensenada, Punta Lara, Cabrera 4897, 617 (ambos LP); Punta Lara, Dawson 952, 377 (ambos LP); Punta Lara, Fabris 7518 (LP). Punta Lara, Ciciarelli 2 (LP). Punta Lara, Atkinson 20738 (SI); Isla Paulino, Marelli 1905 (SI); Dpto. San Fernando, Paraná Miní, Cabrera 10629 (LP). Dpto. Capital, Palermo, Hicken 26937 (SI); Barracas al Sur, Venturi 52 (SI); Dpto. Tigre, Lanfranchi 506 (SI). BRASIL. Prov. Río Grande do Sul: Dpto. Rosario do Sul, Arroyo Chanota, Krapovickas \& Cristóbal 34231 (SI). PARAGUAY. Prov. San Pedro, Dpto. San Pedro, Primavera, Woolston 1034 (SI).

Canna indica L. GUATEMALA. Dpto. Alto Verapaz, Cubilquitz, Von Turckheim 4018 (LIL). PERU: Prov. Loreto, Dpto. Santa María, Allard 22453 (LIL). BRASIL. Prov. Paraná: Dpto. Curitiba, Xaxim, 
Smith \& Reitz 12497 (SI). PARAGUAY. Dpto. Presidente Hayes, Colonia Menno, Misión Nueva Vida, Arenas 1561 (SI). ARGENTINA. Prov. Tucumán: Dpto. Tafí Viejo, orilla de la vía del Tafí al Cadillal, Herrera 345 (LIL); Dpto. Capital, Lillo 51618 (LIL). Río Sali, Meyer 8641 (LIL). Entre Capital y Muñecas, Meyer 134592 (LIL); Dpto. Cruz Alta, Ranchillos, Bailetti 51609 (LIL). Prov. Corrientes: Dpto. Capital, Isla Meza, Rodrigo 837 (LP). Prov. Chaco: Baggi 316 (SI). Prov. Santa Fe: Dpto. Obligado, Tacuarendí, Pire 740 (SI). Prov. Buenos Aires, Dpto. Ensenada, Punta Lara, Dawson 943 (LP). Dpto. La Plata, City Bell, a orillas del arroyo Rodriguez, Ciciarelli 3 (LP).

Canna lineata Ciciar. Bot. Complutensis 38: 115-121. 2014. PARATIPOS. ARGENTINA. Prov. Buenos Aires: Dpto. La Plata, Ringuelet, en zanjón al borde del camino, 511 y 23 Ciciarelli 19 (LP); en pastizal frente a 512 y 22 Bis, Ciciarelli 14 (LP).

Canna paniculata Ruiz et Pav. ARGENTINA. Prov. Misiones, Dpto. Iguazú, salto río Urugua-í, Mulgura et al. 539 (SI); Dpto. Montecarlo, San Vicente a Capitán Morales, $12 \mathrm{Km}$ de San Vicente, Zuloaga et al. 2049 (SI); Dpto. San Javier, Acaraguá (Alto Uruguay), Bertoni 2774, 3682 (ambos LIL); Carpa Cué, Correa et al. 7920 (CTES); Matto Quemado, s. coll. 3706 (LIL). Dpto. General Manuel Belgrano, Bernardo de Irigoyen, Hunziker 925 (LIL); San Antonio, Bertoni 445 (LIL). Dpto. San Pedro, Alegría km. 367, Bertoni 1035 (LIL). Dpto. Iguazú, ruta 12, 20 km al SE de Puerto Iguazú, Ferruci et al. 465 (CTES). Dpto. Oberá, 12 km. Al NE de Panambí s/ ruta 5, Pire 320 (CTES). Dpto. Libertador Gral. San Martín, Puerto Leoní, Crisci 101 (LP). Garuhapé, camino a Gruta del Indio, Piré \& Mroginski 100 (CTES). Prov. Buenos Aires: Dpto. Delta del Paraná, Arroyo Manzano de Medina, Bridarolli 2216 (LP).

Canna tandilensis Ciciar. Bot. Complutensis 39: 87-96. 2015 PARATIPOS. ARGENTINA. Prov. Buenos Aires: Dpto Tandil. Tandil: Monte Calvario, Ciciarelli 20 (LP). URUGUAY. Dpto. Rocha: La Paloma, Ciciarelli 21 (LP).

Canna variegatifolia Ciciar. Rev. Museo de La Plata, Bot. 14 (103): 333-341. PARATIPOS. ARGENTINA. Prov. Buenos Aires: Dpto. La Plata, Calle 44 y rotonda ruta 2, costado del camino, Ciciarelli 12 (LP); Calle 520 y rotonda Av. 25 Ciciarelli 13 (LP); City Bell, Calle 472 e/ Cno. Belgrano y Calle 20, a orillas del Arroyo Rodriguez. Ciciarelli 16 (LP). Prov. Santa Fe: Dpto. La Capital, 27 km Santa al N de la ciudad de Santa Fe, al borde de la ruta 11, Ciciarelli 22 (LP). 